\title{
Low Temperature Hot Filament Chemical Vapor Deposition of Ultrananocrystalline Diamond Films with Tunable Sheet Resistance for Electronic Power Devices.
}

\author{
Jesus J. Alcantar-Peña ${ }^{1,2}$, Jorge Montes ${ }^{2,5}$, M.Josefina Arellano-Jimenez ${ }^{3}$, J. E. Ortega Aguilar ${ }^{3}$, \\ Dainet Berman-Mendoza ${ }^{1}$, Rafael García ${ }^{1}$, M.J. Yacaman ${ }^{3}$, Orlando Auciello ${ }^{2,4, *}$ \\ ${ }^{1}$ Departamento de Investigación en Física, Universidad de Sonora, Hermosillo, Sonora, 83000, México. \\ ${ }^{2}$ Materials Science and Engineering, University of Texas-Dallas, Richardson, TX 75080, USA \\ ${ }^{3}$ Physics and Astronomy, University of Texas-San Antonio, San Antonio, TX 78249, USA \\ ${ }^{4}$ Bioengineering, University of Texas at Dallas, Richardson, TX 75080, USA \\ ${ }^{5}$ Departamento de Física, Universidad de Sonora, Hermosillo, Sonora, 83000, México \\ * Corresponding author: orlando.auciello@utdallas.edu
}

\begin{abstract}
This paper describes the results from systematic experiments performed to investigate the influence of different substrate-filaments distances, surface-substrate temperatures and reaction of precursors during the growth of Ultrananocrystalline Diamond (UNCD) films via the hot filament chemical vapor deposition (HFCVD) process. The experimental results provide valuable information to understand the important role of key molecules $\left(\mathrm{CH}_{\mathrm{x}} \mathrm{x}=1,2,3\right)$ and atoms $(\mathrm{H})$, resulting from the cracking of precursor molecules $\left(\mathrm{CH}_{4}, \mathrm{H}_{2}\right)$ on the hot surface of the filaments, and the contribution of argon (Ar) inert gas atoms, all interacting on the surface of the substrates when using different filaments-substrate distances to produce films with different structures and properties. The interaction of the cracked molecular and atomic species at different filamentssubstrate distances, play a critical role in the nucleation and growth of films with different structures, as observed by complementary analytical techniques. Films grown at 5, 15, and $30 \mathrm{~mm}$ filaments-substrate distances exhibit graphite-disordered graphene phases, while those grown at $20 \mathrm{~mm}$ distances exhibit mixed large UNCD-minor graphite mixed phases, and those grown at 25 and $35 \mathrm{~mm}$ distances exhibit pure UNCD phase. UNCD films grown at $\sim 600{ }^{\circ} \mathrm{C}$ exhibit grain boundaries with $\mathrm{sp}^{2}$ and Trans-Polyacetylene (TP-A) dangling chemical bonds and low sheet resistance $(\sim 5 \Omega)$, while UNCD films grown at relativity low temperature $\left(\sim 490{ }^{\circ} \mathrm{C}\right)$ exhibit high sheet resistance $(\sim 8 \mathrm{M} \Omega)$ and more TP-A dangling bonds. The results presented in this paper indicate that UNCD and mixed UNCD/disordered graphene/graphite films can be grown with tailored resistivity enabling potential applications in electronic power devices.
\end{abstract}




\section{Introduction}

Diamond films have been attracting the interest of the research and technology-oriented communities because they exhibit a unique combination of properties such as high wear resistance, highest hardness relative to any other film, lowest friction coefficient compared with metal and ceramic coatings, chemical inertness, excellent thermal conductivity $\left(\sim 1500 \mathrm{~W} /{ }^{\circ} \mathrm{K} . \mathrm{m}\right.$, close to that of single crystal diamond $\sim 2100 \mathrm{~W} /{ }^{\circ} \mathrm{K} . \mathrm{m}$ ) perpendicular to the film surface and in the film plane for microcrystalline diamond ( $\geq 1 \mu \mathrm{m}$ grain size) , tunable electronic properties by doping, and biocompatible properties. Extensive fundamental and applied science on UNCD films growth has resulted in applications to diverse range of devices [1-4].

Microwave Plasma Chemical Vapor Deposition (MPCVD) [1] and Hot Filament Chemical Vapor Deposition (HFCVD) [5-7] are the two techniques being most used to grow diamond films with various microstructures $[1,5-7]$ and at different substrate temperatures $[1,5-7]$. UNCD films exhibit high electrical conductivity with either nitrogen incorporated in grain boundaries or boron doping via substitution of $\mathrm{B}$ for $\mathrm{C}$ atoms in the diamond lattice [1]. Furthermore, UNCD films also exhibits outstanding biocompatibility [1, 8-10]. All the UNCD film properties mentioned above enable a broad range of applications to a new generation of mechanical, electronic, energy generation and bio-medical devices.

One topic that has been substantially explored is the UNCD film growth mechanism both for the MPCVD and HFCVD processes. For the HFCVD process, there are various issues that are still under discussion, such as, precursor's reaction at different traveling distance from the moment of their cracking at the hot filaments' surface until the arrival on the substrate's surface to nucleate and induce the growth of the films. The understanding of precursor's reaction during the pathway from the filament towards the substrate surface may be critical to understand the range of carbon 
faces observed in the films, like disordered graphite/graphene mixtures and UNCD phases with mixtures of sp² and TP-A C-atoms bonding in the films' grain boundaries. The precursor's reaction may contribute to chemical bonds and resistivity of UNCD films may enable applications of these films in a new produce $\mathrm{UNCD}$ films at relativity low $\left(\sim 270^{\circ} \mathrm{C}[6] 490{ }^{\circ} \mathrm{C}[7]\right)$ or higher $(\sim 600$ $\left.{ }^{\circ} \mathrm{C}\right)$ temperatures, with high and low resistivity, respectively. The feasibility of tuning grain boundaries with dangling generation of diamond-based electronic power devices. One relevant characteristic of polycrystalline diamond films for electronic device applications may be their hydrogenated 2D surface conductivity, which may be provided by chemical dangling bonds on the surface [11-13]. In this respect, by changing the filament-substrate distance, it may be possible to change the nature of the chemical bonds on the surface and grain boundaries of the UNCD films, thus affecting their electrical resistivity, which can provide a pathway for application of UNCD films to the fabrication of diamond-based power devices.

\section{Experimental}

Because of substrates exchange insertion into the HFCVD film growth chamber, through a load lock, it is not practical to place a thermocouple on the substrate surface every time. Thus, the substrate surface temperature cannot be measured directly during every single film growth. Therefore, a substrate surface temperature calibration was performed before film growth. The temperature calibration was performed using a K-type thermocouple in direct contact with the substrate's surface to measure the substrate surface temperature while exposing it to identical conditions as during film growth. A typical calibration curve correlating the substrate surface temperature and sample holder temperatures applying power in the heater device with filaments off is shown in Fig. 1a. In addition, Fig. 1b shows the calibrated substrate surface temperature for all filament-substrate distances and with filaments on $\left(2200^{\circ} \mathrm{C}\right)$, used for growing all carbon-based 
films investigated. Figure 1c shows the temperature distribution in the space between the filaments and the substrates for the different substrate-filaments distance used to grow the films, calculated using the COMSOL 4.2 software. This temperature distribution is critically related to the substrate surface temperature achieved at the different filament-substrate surface distance used to grow all the films described in this paper.
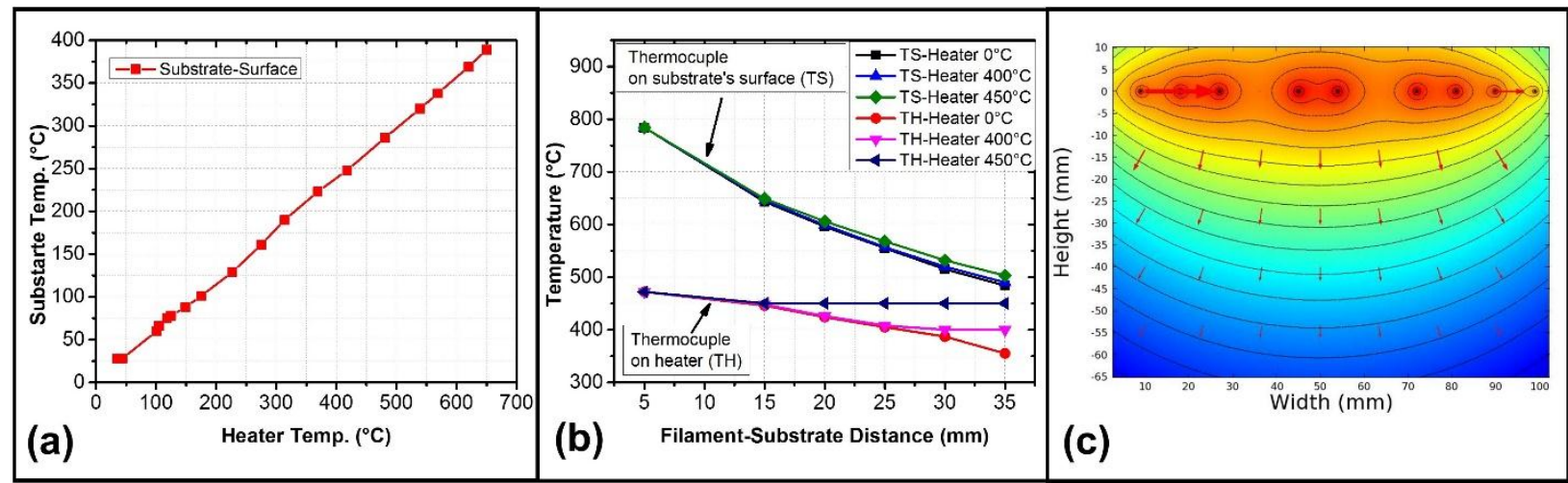

Figure 1. a) Curve corresponding to the surface-substrate temperature calibration performed to determine the substrate surface temperature during film growth, from reading the temperature provided by the substrate surface-connected thermocouple and the substrate holder-connected thermocouple; b) Substrate surface temperature vs. filament-substrate distance; c) Temperature distribution in the space between the filaments and the substrates for the different substratefilaments distance used to grow the UNCD films, calculated using the COMSOL 4.2 software.

The HFCVD system features and arrangement of 8 parallel and two angled tungsten (W) filaments $(\varnothing=0.25 \mathrm{~mm}$, length $\sim 14.2 \mathrm{~cm}$ ) (Fig. 2b) held on a Molybdenum (Mo) frame positioned above the substrate holder (Figs. 2a and 2c). The distance between the filaments and the substrate surface was changed by moving the substrate using a motorized Z-driving system (Fig. 2a) provided by the HFCVD manufacturer. The filaments-substrate distances used in the experiments described here were 5, 15, 20, 25, 30 and $35 \mathrm{~mm}$. 


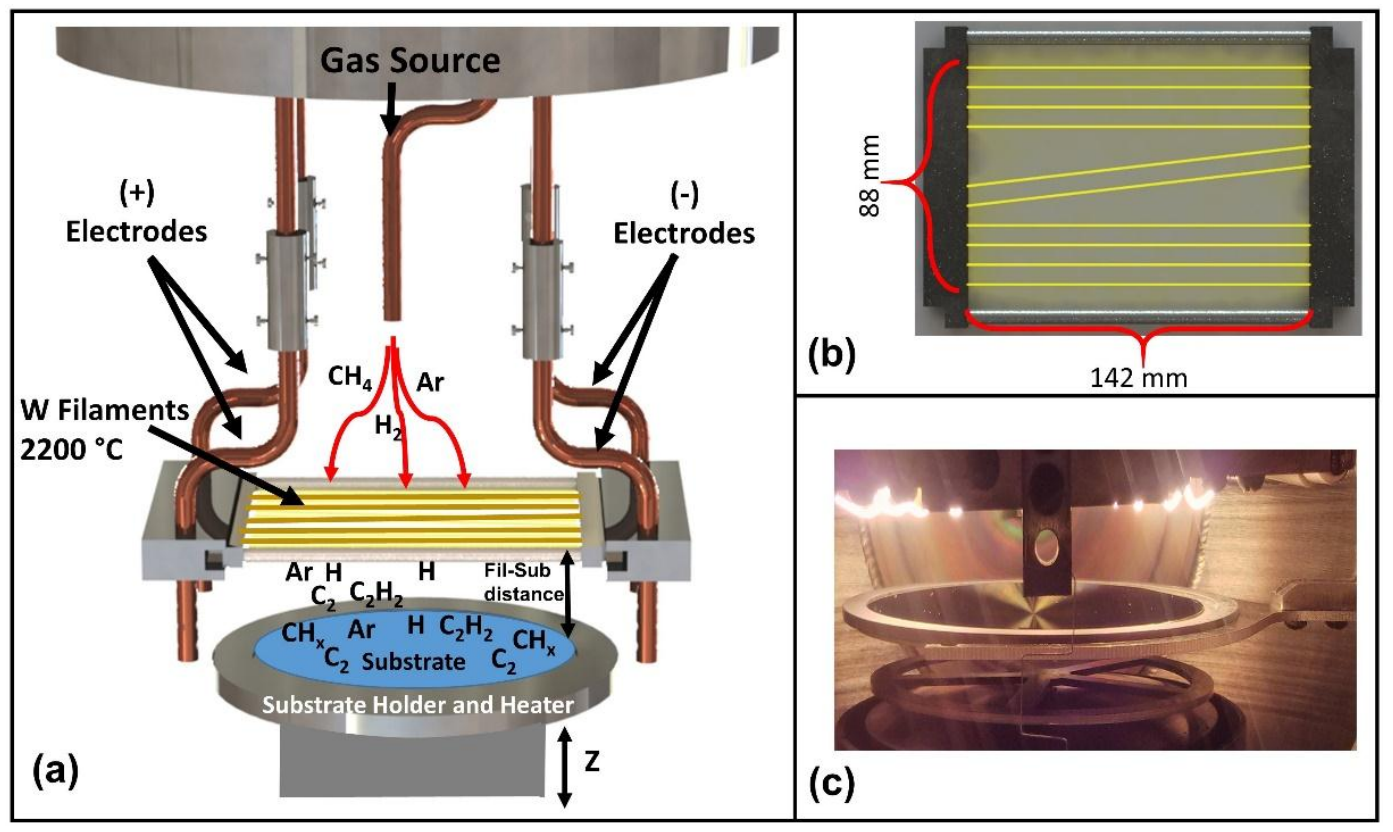

Figure 2. a) Schematic of the HFCVD-substrate geometrical arrangement and gas flow and species involved in the UNCD film growth process via the HFCVD system used in the experimental work described here; b) schematic of the filament arrangement (the specific filament arrangement shown in the figure is the one that provides the best film thickness uniformity in conjunction with the appropriate filament-substrate distances of 25, 30, and $35 \mathrm{~mm}$ (see Table 1), across $100 \mathrm{~mm}$ diameter substrates, compared with UNCD films grown with other filament arrangements); c) picture of the HFCVD system at the time of transferring a substrate into the growth chamber from the load-lock (the rotating holder on which the substrate holder is positioned can be seen under the substrate holder with the wafer) .

The substrates were heated by radiation from the filament plus extra heating provided by heating the substrate heater to $400^{\circ} \mathrm{C}$ and $450^{\circ} \mathrm{C}$, as measured by the thermocouple in contact with the heater surface. The substrates were continuously rotated during film growth to provide a uniform temperature and carbon radical species homogeneous distribution on the substrate surface. All films were grown for $2 \mathrm{hrs}$. for each experiment, in order to have the time parameter constant for all films. Computer simulations were performed, using a COMSOL 4.2 software, to analyze 
the temperature distribution in the space between the filaments and the substrates for the different substrate-filaments distance used to grow the films (Fig. 1c).

The filaments were heated to $2200{ }^{\circ} \mathrm{C}$ by passing a DC current during the film growth. Films were grown using gas flows of $\mathrm{H}_{2}(5 \mathrm{sccm}), \mathrm{CH}_{4}(1 \mathrm{sccm})$ and $\mathrm{Ar}(45 \mathrm{sccm})$. More details of the UNCD film growth process can be found in our prior paper [7]. The surface morphology, films thickness and nanostructure were characterized using scanning electron microscopy (SEM, ZEISS SUPRA-40) and high-resolution transmission electron microscopy (HRTEM, JEOL 2010F). The samples for the HRTEM analysis were prepared using a focused ion beam (ZEISS FIB-SEM CROSSBEAM) process. The crystallinity and grain sizes of UNCD films were investigated via complimentary HRTEM with electron -diffraction and X-ray diffraction (XRD, Rigaku Ultima III, $\mathrm{Cu} K \alpha$ radiation, $1.542 \AA$ ). The type of carbon chemical bonding in the films were analyzed via visible Raman spectroscopy, using a Thermo DXR Raman spectrometer with $532 \mathrm{~nm}$ wavelength laser beam. The sheet resistance of the UNCD films was measured using an Alessi 4point probe system with $\mathrm{C} 4 \mathrm{~S}$ probe head with $1 \mathrm{~mm}$ spacing between the tips.

\section{Results and Discussion}

Success in producing continuous and dense UNCD or mixed UNCD-graphite or UNCDgraphite/disordered graphene films at different temperatures, with tailored chemical bonds in grain boundaries and tunable sheet resistance, has important implications for application of these films in electronic power devices like Thin Film Transistors (TFTs) or Schottky diodes, based on nanocarbon films with hydrogenated surface. Figure 3 shows Raman analysis (using visible 532 $\mathrm{nm}$ laser wavelength at $1 \mathrm{~mW}$ of power) of films grown at substrate surface temperatures in the range $490-800{ }^{\circ} \mathrm{C}$ for filament-substrate distances of $5 \mathrm{~mm}$ to $35 \mathrm{~mm}$ (Figs. 3a and $3 \mathrm{~b}$ ). The temperature of the substrate surface was reached by the addition of the filament radiation at 
different filament-substrate distances plus additional heating from the holder heater to $400{ }^{\circ} \mathrm{C}$ (Fig. 3a) and $450^{\circ} \mathrm{C}$ (Fig. 3b). It is important to emphasize that all films, for which Raman analyses are shown in Fig. 3, were grown at different substrate-filaments distances, as indicated in that figure.

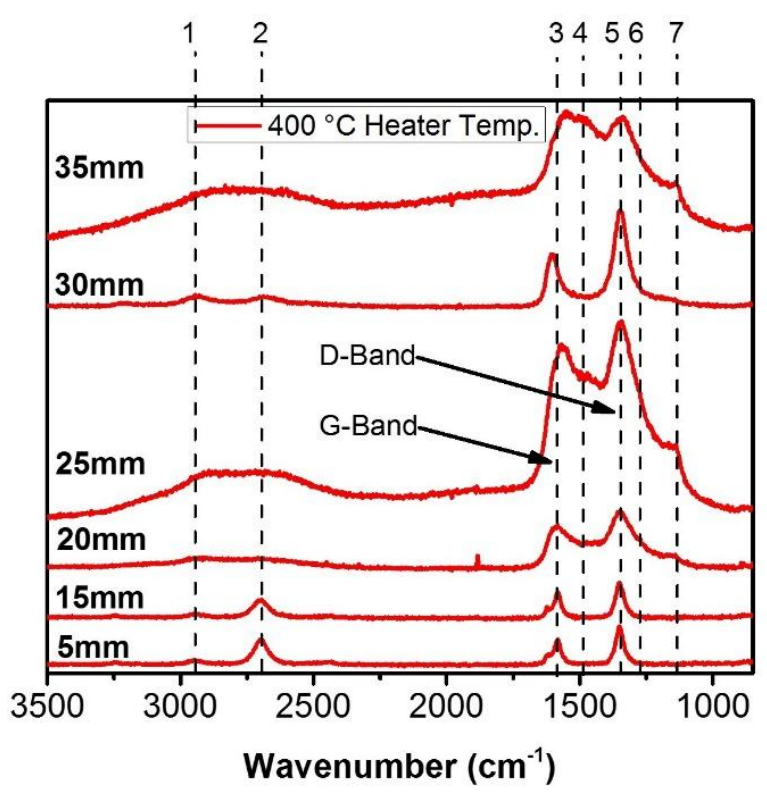

(a)

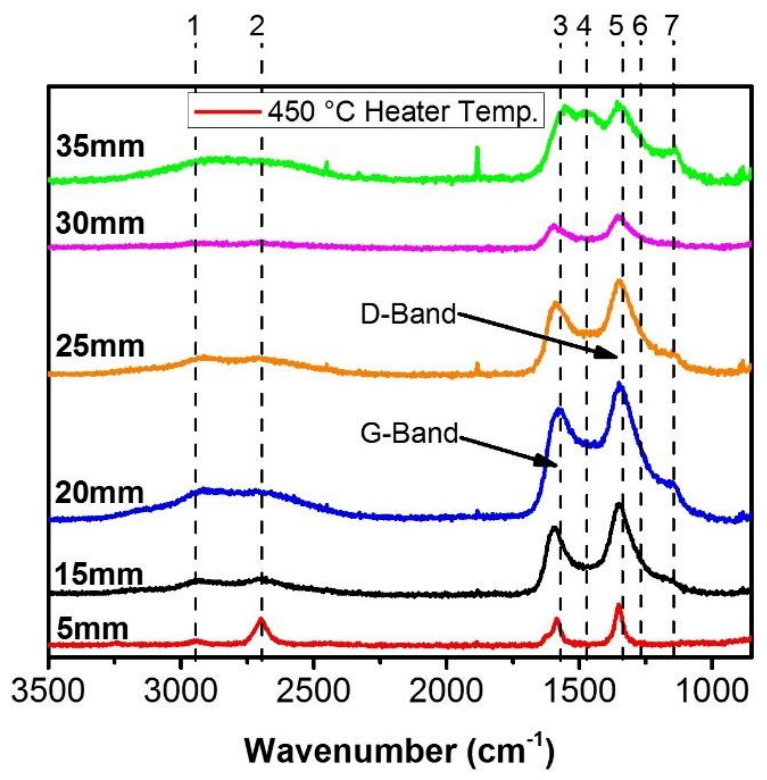

(b)

Figure 3. Raman spectra of films grown at filament-substrate distances from $5 \mathrm{~mm}$ to $35 \mathrm{~mm}$ with the substrate heater heated to $400{ }^{\circ} \mathrm{C}$ (a) and $450{ }^{\circ} \mathrm{C}$ (b), respectively, read by the thermocouple in contact with the substrate holder surface.

The contributions of the heat supplied by the filament radiation, the substrate positions, the heat supplied by the substrate holder heater and the reaction of the precursors at different traveling distance from the moment of precursor's cracking at the hot filaments surface, until the nucleation and film growth on the substrate surface, can be seen from the Raman analysis. The spectra show characteristic D band $\left(\sim 1340 \mathrm{~cm}^{-1}\right)$ and $\mathrm{G}$ band $\left(\sim 1588 \mathrm{~cm}^{-1}\right)$, peaks 5 and 3 , respectively, from the $\mathrm{sp}^{2} \mathrm{C}$-atoms bond hybridization. Deconvolution of the Raman peaks (not shown to simplify the spectrum figure) of the UNCD films shows that there is a contribution of three extra peaks between 1000-2000 $\mathrm{cm}^{-1}$, peak $4\left(\sim 1489 \mathrm{~cm}^{-1}\right)$, peak $6\left(\sim 1258 \mathrm{~cm}^{-1}\right)$ and peak $7\left(\sim 1164 \mathrm{~cm}^{-1}\right)$, to the $\mathrm{D}$ and $\mathrm{G}$ peaks, reported as representative of $\mathrm{C}=\mathrm{C}$ stretching of Trans-PolyAcetillene (T-PA), 
broadened vibrational density of states (VDOS) of small diamond clusters, and tetrahedral amorphous carbon, and T-PA C-H bending combined with C-C stretching, respectively. [14-17] In addition, the wavenumber range of 3500-2500 $\mathrm{cm}^{-1}$ include peaks $1\left(\sim 2947 \mathrm{~cm}^{-1}\right)$ and $2(\sim 2700$ $\mathrm{cm}^{-1}$ ), which are D+G-mode and 2D-mode respectively.

At the nearest substrate-filaments distance $(5 \mathrm{~mm})$, and due to the high filament radiation heating, the films are grown with the substrate surface at $\geq 800{ }^{\circ} \mathrm{C}$. The films grown at this high temperature show Raman peaks characteristic of structural disordered graphene $\left(2 \mathrm{D}\left(2,700 \mathrm{~cm}^{-1}\right)\right.$ and $\mathrm{D}\left(1,340 \mathrm{~cm}^{-1}\right)$ band peaks), with the graphene structure, seen also in other HFCVD growth [18], confirmed by HRTEM (not shown). The results described above on change of the diamond phase to graphite or disordered graphene, are in agreement with prior studies, which showed that the diamond structure is damaged or changed into other carbon structures other than diamond when it is exposed to high temperatures $\left(\geq 800{ }^{\circ} \mathrm{C}\right)[19,20]$. Raman analysis revealed structural changes in the films grown at $15-35 \mathrm{~mm}$ filament-substrate distances when supplying heat to the substrate surface from the substrate heater, in addition to the heating supplied by the filament radiation (Figs. 3a-b). Specifically, for films grown at 5 and $15 \mathrm{~mm}$ filament-substrate distance, mainly a structural disordered graphene was observed, as revealed by Raman analysis for the film grown with substrate holder heater heated to $400{ }^{\circ} \mathrm{C}$ (confirmation by HRTEM-not shown), and a mixture of graphite and UNCD was detected for the films grown with the substrate heater heated to $450{ }^{\circ} \mathrm{C}$ (substrate surface at $\sim 650^{\circ} \mathrm{C}$-see Fig. 3b, with confirmation by HRTEM-Fig. 4a). The conclusions presented above, from the Raman analysis, are based on the observation of the high intensity and shape of the characteristic $\mathrm{D}$ band $\left(1340 \mathrm{~cm}^{-1}\right)$ and $\mathrm{G}$ band $\left(1588 \mathrm{~cm}^{-1}\right)$ peaks from the $\mathrm{sp}^{2}$ hybridization, and very low signal from the three extra peaks at $1489 \mathrm{~cm}^{-1}, 1258 \mathrm{~cm}^{-1}$ and 1164 $\mathrm{cm}^{-1}$ known as $\mathrm{C}=\mathrm{C}$ stretching of Trans-PolyAcetillene (T-PA), broadened vibrational density of 
states (VDOS) of small diamond clusters and tetrahedral amorphous carbon, and T-PA C-H bonding combined with C-C stretching, respectively [14-16, 20]. For films grown at 20 and 25 mm filament-substrate distance, the characteristic visible Raman spectrum of UNCD films was observed for films grown with the substrate holder heater set up at $400{ }^{\circ} \mathrm{C}$ and $450{ }^{\circ} \mathrm{C}$, respectively (Figs. 3a-b, confirmed by HRTEM-Figs. 4b-c). At $30 \mathrm{~mm}$ filament-substrate distance, the films showed a graphitic phase mixed with UNCD for films grown both with theater set up at $400{ }^{\circ} \mathrm{C}$ and $450{ }^{\circ} \mathrm{C}$ (Figs. 3a-b, confirmed by XRD analysis). Finally, films grown at $35 \mathrm{~mm}$ filamentsubstrate distance with similar heater and substrate surface temperature as for the case of film grown at $30 \mathrm{~mm}$ filament-substrate distance, exhibit the characteristic UNCD structure, as revealed by visible Raman analysis (Figs. 3a-b, confirmed by HRTEM-Fig. 4d).

The UNCD films grown at $35 \mathrm{~mm}$ filament-substrate distance exhibit an intense signal from T-PA dangling bonds at grain boundaries and particularity they were produced at relativity low substrate surface temperature $\left(\sim 490{ }^{\circ} \mathrm{C}\right)$, increasing the substrate compatibility. Similar UNCD films grown at about $500{ }^{\circ} \mathrm{C}$ on Si substrates were observed by X. Xiao et all [2], using the MPCVD process. The heat transfer simulation by COMSOL at different substrate-filaments distances, shown in Fig. 1c, indicate that at close distance (0-15 $\mathrm{mm}$ height) to the filaments, the temperature radiation it's mainly driven by the number and position of the filaments, and the temperature fluctuations correlate with the film thickness fluctuations shown in Table $\mathbf{1 .}$

The structures of key films were analyzed by HRTEM, as shown in Figure 4, for films grown at $15 \mathrm{~mm}$ (a), $20 \mathrm{~mm}(\mathrm{~b}), 25 \mathrm{~mm}$ (c) and $35 \mathrm{~mm}$ (d) filament-substrate distance at $450^{\circ} \mathrm{C}$ heater temperature for all films. The diffraction patterns show diffraction rings related to Van der Waals and covalent bond distance corresponding to the graphite structure in the mixed graphite-UNCD film structure in Fig. 4a, and the diffraction peaks and rings corresponding to the (111) and (220) 
diamond planes in Fig. 4a and the UNCD films in Figs. 4b-d. The crystallinity characteristic of the diamond phase in the UNCD films, determined by electron diffraction patterns, was confirmed by XRD analysis (see Fig. 6 and Table 2).

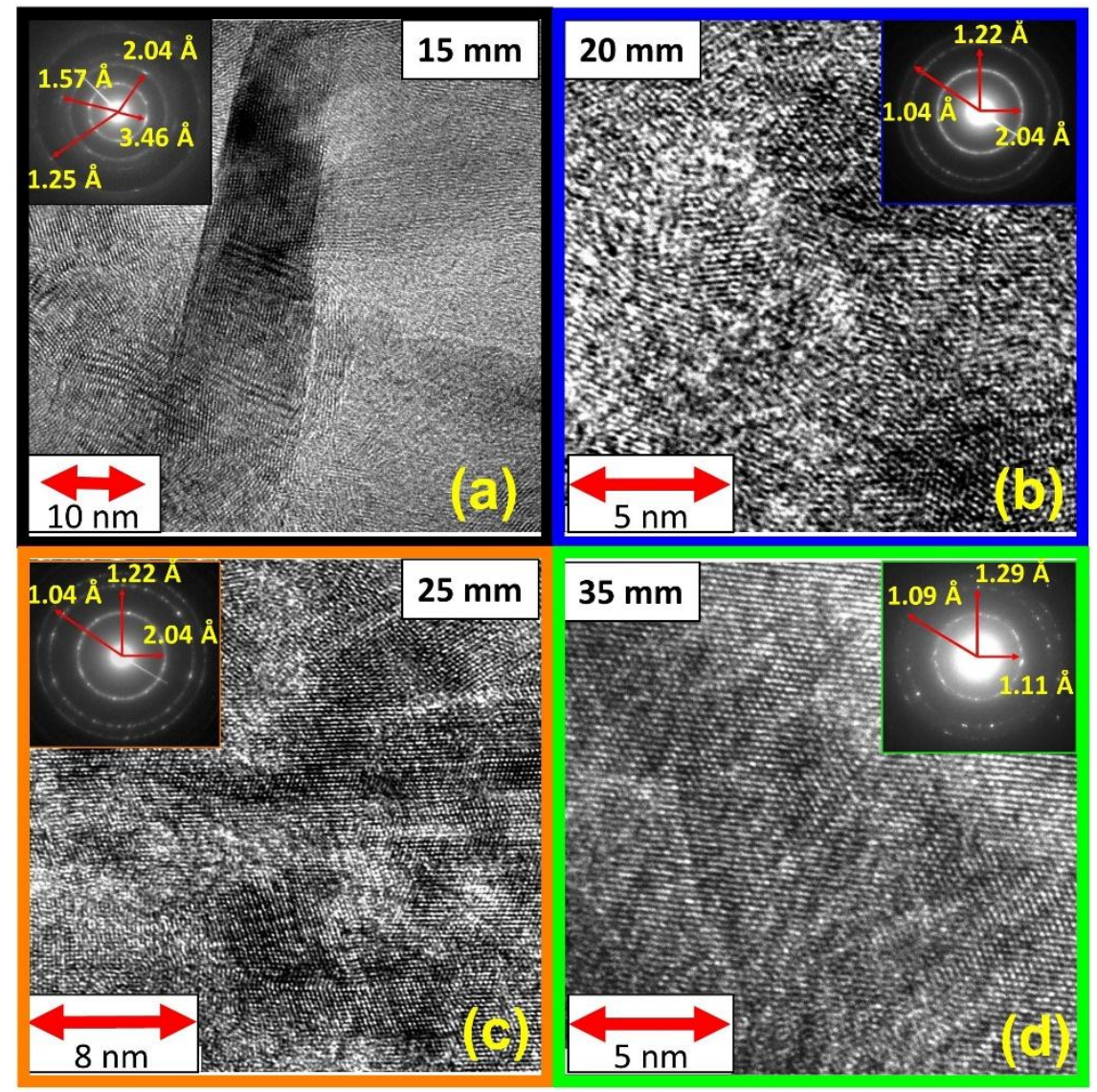

Figure 4. HRTEM images from films grown at different filament-substrate distance and $450{ }^{\circ} \mathrm{C}$ heater temperature: (a) $15 \mathrm{~mm}$ (large graphite-small UNCD mixture), (b) $20 \mathrm{~mm}$, (c) $25 \mathrm{~mm}$, (d) $35 \mathrm{~mm}$ (UNCD films), correlating with Raman spectra shown in Fig. 3b (match the frame colors in this figure with the spectra color in Fig. 3b).

Figure 5 shows top view SEM pictures of the morphologies of thin films grown at different filament-substrate distances and substrate temperatures, namely: (a) films grown with heater at $400{ }^{\circ} \mathrm{C}$ and (b) heater at $450^{\circ} \mathrm{C}$. The characteristics of the films are explained in the figure caption. 


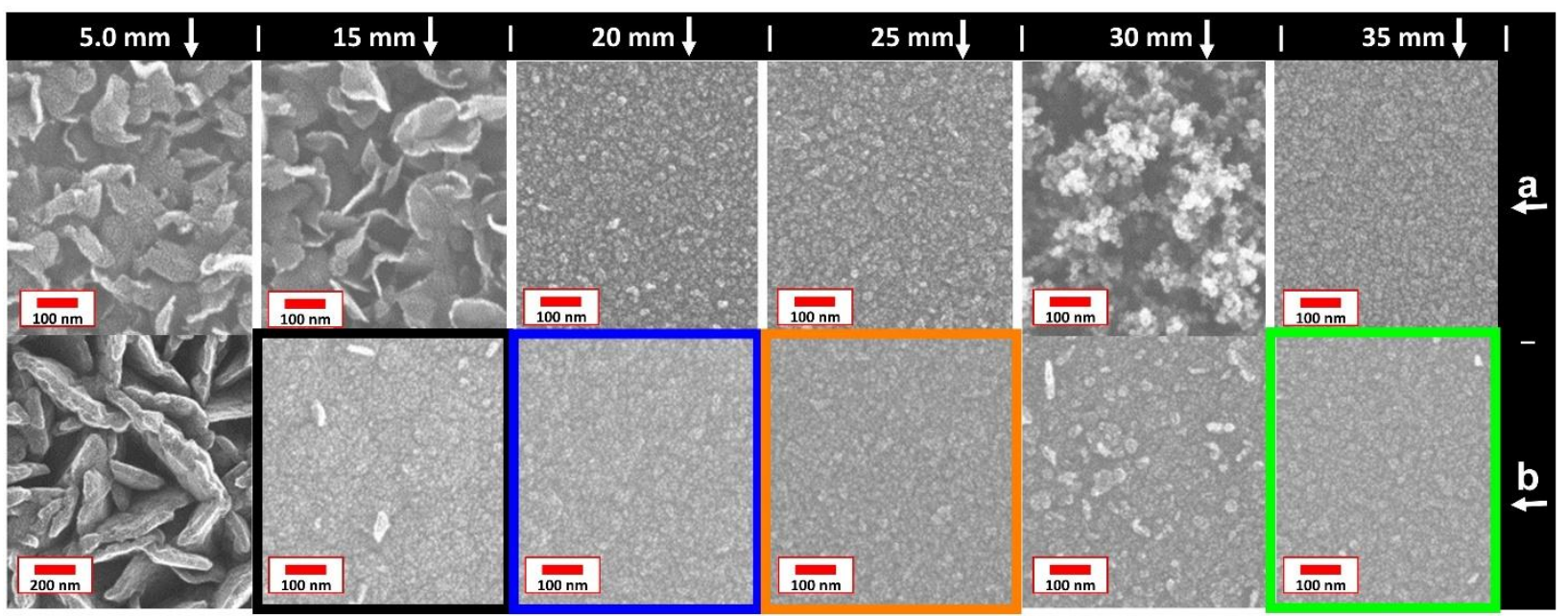

Figure 5. SEM top view images of the films grown at different filament-substrate distances and substrate surface temperatures: a) films grown with the heater at $400{ }^{\circ} \mathrm{C}$ and b) with the heater at 450 ${ }^{\circ} \mathrm{C}$. The SEM pictures of the surface of the films grown at 20, 25, and $35 \mathrm{~mm}$ filament-substrate distance for both substrate heater heated to $400{ }^{\circ} \mathrm{C}$ and $450{ }^{\circ} \mathrm{C}$ show the topography characterized by the nanostructure of UNCD films; the SEM pictures of films grown at 5, 15, and $30 \mathrm{~mm}$ filamentsubstrate distances with the heater at $400{ }^{\circ} \mathrm{C}$ and $5 \mathrm{~mm}$ with the heater at $450{ }^{\circ} \mathrm{C}$ show the characteristic surface morphology of graphite; the films grown at 15 and $30 \mathrm{~mm}$ filament-substrate distances with the heater at $450{ }^{\circ} \mathrm{C}$ show a surface morphology of mixed UNCD-graphite phases (match the frame colors in this figure with the spectra color in Fig. 3b).

Table 1 shows all the film thickness measurements from SEM cross sections (not shown) obtained at different wafer positions for films grown with different heater temperatures (substrate surface temperatures) and different substrate-filaments distances. The larger film thickness variations across the film surface, observed for films grown at 5 and $15 \mathrm{~mm}$ filament-substrate distance, are mainly driven by the number and position of the filaments at closer distances due to thermal radiation effects as described above (see Fig. 1c). 


\begin{tabular}{ccc}
\hline $\begin{array}{c}\text { Distance } \\
\mathbf{m m}\end{array}$ & $\begin{array}{c}\text { Thickness (nm) } \\
\text { Heater at } \mathbf{4 0 0}{ }^{\circ} \mathbf{C} \\
\text { Center | Middle | Edge }\end{array}$ & $\begin{array}{c}\text { Thickness (nm) } \\
\text { Heater at 450 }{ }^{\circ} \mathbf{C} \\
\text { Center | Middle | Edge }\end{array}$ \\
\hline 5 & $989.2-1209-565.2$ & $1512.3-1227.3-1686.3$ \\
15 & $322.7-334.2-134.0$ & $325-325.4-240.4$ \\
20 & $177.9-147.7-76.61$ & $419.1-386.8-245.1$ \\
25 & $277.2-248.0-76.6$ & $171.5-154.2-114.2$ \\
30 & $239.2-221.5-154.2$ & $251.7-220.3-180.2$ \\
35 & $81.4-81.8-54.6$ & $137.0-118.5-78.3$ \\
\hline
\end{tabular}

Table 1. UNCD film thickness as obtained from SEM cross section measurements at different positions across the films on $100 \mathrm{~mm}$ diameter substrates for films grown with different substrate temperatures and different substrate-filaments distances. The better uniformity observed for UNCD films grown at 25, 30, and $35 \mathrm{~mm}$ filament-substrate distance correlates with the better heat distribution across the filaments length as revealed by the calculations shown in Fig. 1c.

The importance of performing analysis with complimentary techniques is revealed by the fact that in the electron diffraction analysis of the films grown at $15 \mathrm{~mm}$ filament-substrate distance (Fig. 4a) the evidence of the existence of a minor diamond phase mixed with the major graphitic phase observed is confirmed in the XRD analysis of the same film. The XRD analysis for a film grown at 5 and $15 \mathrm{~mm}$ filament-substrate distance shows a major peak ( 1 at $\left.26.54^{\circ}\right)$ corresponding to the graphitic (002) phase (black and red spectrums - Fig. 6) and also small peaks ( 4 at $\sim 44.01^{\circ}$, corresponding to diamond (111), 7 at $75.44^{\circ}$, corresponding to diamond (220) and 8 at $91.70^{\circ}$, corresponding to diamond (311)). These diamond peaks are the dominant peaks in the XRD spectra of the UNCD films grown at 20 (blue curve), 25 (brown curve), and 35 (violet curve) mm filamentsubstrate distance (Fig. 6). For the case of $30 \mathrm{~mm}$ (green curve), a balanced mixture of graphite and UNCD phases was observed. The XRD analysis shows excellent agreement with the HRTEM images and electron diffraction analysis shown in Figs. 4b-d and the Raman analysis of the same 
films shown in Figs. 3a-b. In addition to the XRD peaks corresponding to the graphitic and diamond phases, peaks 2 and 3 at $34.76^{\circ}$ and 39.51 , respectively, correspond to tungsten carbide from the interface between the UNCD film and the tungsten layer on the Si substrate on which the UNCD films were grown on the carbide nucleation layer. Finally, the peaks 5 and 6 at $54.45^{\circ}$ and $61.80^{\circ}$ correspond to Tungsten from the tungsten layer grown on the Si substrate.

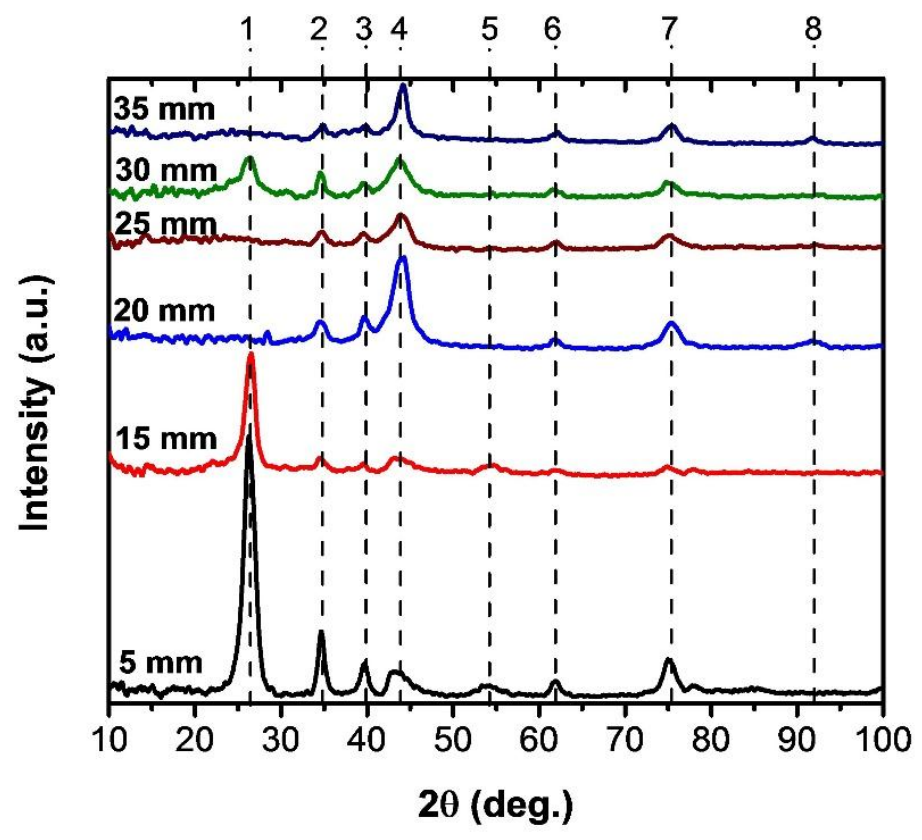

Figure 6. (XRD spectra from analysis of films grown at $5 \mathrm{~mm}, 15 \mathrm{~mm}, 20 \mathrm{~mm}, 25 \mathrm{~mm}, 30 \mathrm{~mm}$ and $35 \mathrm{~mm}$ filament-substrate distance at $450{ }^{\circ} \mathrm{C}$ heater temperature. The black and red spectra show the dominant graphite peak (1) and very small diamond peaks $(4,7$, and 8$)$ for the film grown at 5 and $15 \mathrm{~mm}$ filament-substrate distance. The blue, brown and violet spectra show the dominant diamond peaks $(4,7$, and 8 ) characteristic of high quality UNCD films; the green spectra shows a balanced mixture of graphite and UNCD phases.

Diffraction angles, lattice spacing, grain size and lattice constant from films grown at $5 \mathrm{~mm}$, $15 \mathrm{~mm}, 20 \mathrm{~mm}, 25 \mathrm{~mm}, 30 \mathrm{~mm}$ and $35 \mathrm{~mm}$ at $450^{\circ} \mathrm{C}$ heater temperature substrate surface temperature are summarized in Table 2. 


\begin{tabular}{ccccccc}
\hline $\begin{array}{c}\text { Distance } \\
\mathbf{m m}\end{array}$ & $\begin{array}{c}\text { Position } \\
\left({ }^{\circ} \mathbf{2} \boldsymbol{\theta}\right)\end{array}$ & $\begin{array}{c}\text { Miller Indices } \\
(\mathbf{h k l})\end{array}$ & $\begin{array}{c}\text { d-spacing } \\
\mathbf{d}(\mathbf{A})\end{array}$ & $\begin{array}{c}\text { Grain Size } \\
\mathbf{n m}\end{array}$ & $\begin{array}{c}\text { Lattice } \\
\text { Constant(Å) }\end{array}$ & Material \\
\hline 5 & 26.25 & 002 & 3.3923 & 5.64 & 6.7845 & Graphite \\
15 & 26.54 & 002 & 3.3558 & 6.55 & 6.7117 & Graphite \\
30 & 26.33 & 002 & 3.3821 & 4.77 & 6.7643 & Graphite \\
5 & 43.25 & 111 & 2.0902 & 3.43 & 3.6204 & Diamond \\
15 & 43.38 & 111 & 2.0842 & 3.06 & 3.6101 & Diamond \\
20 & 44.01 & 111 & 2.0559 & 4.36 & 3.5609 & Diamond \\
25 & 44.08 & 111 & 2.0527 & 4.30 & 3.5556 & Diamond \\
30 & 43.73 & 111 & 2.0684 & 3.81 & 3.5826 & Diamond \\
35 & 44.17 & 111 & 2.0488 & 6.78 & 3.5487 & Diamond \\
5 & 75.03 & 220 & 1.2649 & 6.86 & 3.5777 & Diamond \\
15 & 74.94 & 220 & 1.2662 & 7.87 & 3.5814 & Diamond \\
20 & 75.34 & 220 & 1.2605 & 4.85 & 3.5652 & Diamond \\
25 & 75.18 & 220 & 1.2628 & 4.50 & 3.5716 & Diamond \\
30 & 75.02 & 220 & 1.2651 & 5.65 & 3.5781 & Diamond \\
35 & 75.44 & 220 & 1.2589 & 5.62 & 3.5607 & Diamond \\
20 & 91.70 & 311 & 1.0728 & 4.60 & 3.5582 & Diamond \\
25 & 92.09 & 311 & 1.0701 & 13.08 & 3.5487 & Diamond \\
35 & 91.79 & 311 & 1.0727 & 10.09 & 3.5579 & Diamond \\
\hline & & & & & &
\end{tabular}

Table 2. Diffraction angles, lattice spacing, grain size and lattice constant from films grown a $5 \mathrm{~mm}, 15 \mathrm{~mm}, 20 \mathrm{~mm}, 25 \mathrm{~mm}, 30 \mathrm{~mm}$ and $35 \mathrm{~mm}$ filament-substrate distances at $450^{\circ} \mathrm{C}$ heater temperature.

The observed variations in the diamond lattice d-spacing correspond to residual stress on the films. The XRD analysis shows that the d-spacing in the diamond lattice, calculated from Bragg's law, is decreasing from $2.0559 \AA$ for the film grown at $20 \mathrm{~mm}$ filament-substrate distance to $2.0488 \AA$ for the film grown at $35 \mathrm{~mm}$ filament-substrate distance, which relates to a reduction of the lattice constant from $3.5609 \AA$ to $3.5487 \AA$, thus revealing that the UNCD films are under compressive stress $[17,21]$. The comparison of the XRD and HRTEM analysis of the films grown at $15 \mathrm{~mm}$ filament-substrate distance shows excellent agreement in confirming that the films structure correspond to a diamond phase immersed in a graphitic matrix, which is the dominant material phase in the films. 
Figure 7 shows the sheet resistance of films grown at different heater temperature/substrate surface temperature and substrate-filaments distances.

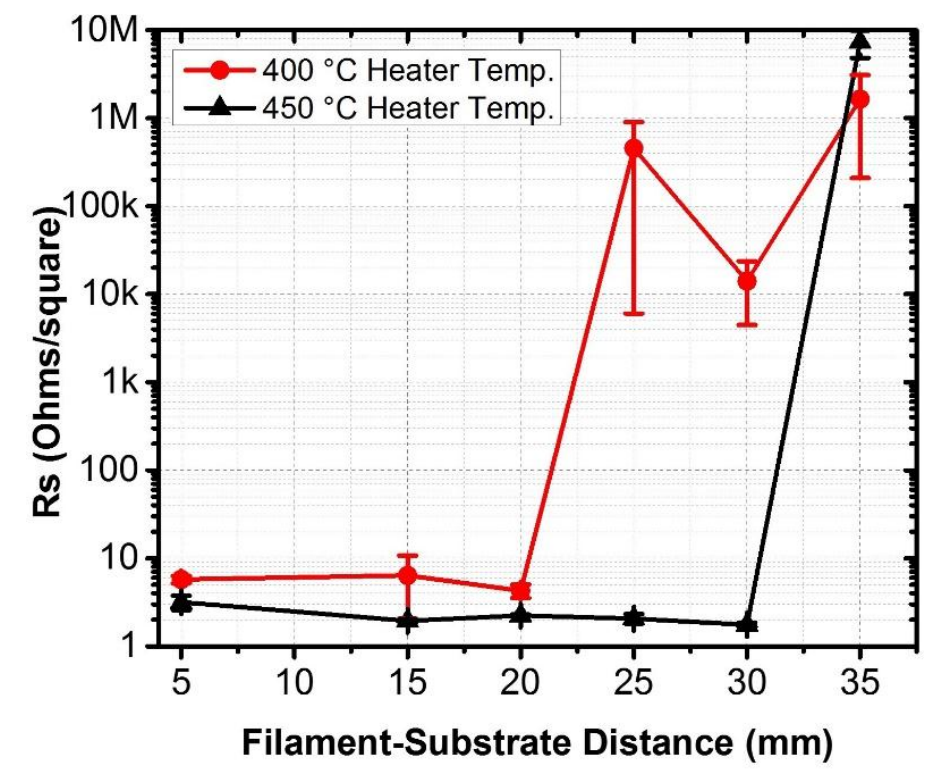

Figure 7. Sheet resistance of UNCD films grown at 400 and $450{ }^{\circ} \mathrm{C}$ heater temperature at different filaments-substrate distance.

The data presented above provides good information showing the dominant high resistivity diamond phase in the UNCD films grown at 25 and $35 \mathrm{~mm}$ filament-substrate distance and $400{ }^{\circ} \mathrm{C}$ heater temperature, and the low resistivity mixed graphite-diamond phase in the films grown at 15, 20 and $30 \mathrm{~mm}$ filament-substrate distance and $450{ }^{\circ} \mathrm{C}$ heater temperature. However, in order to understand how the structural phase-change in the films affect the electrical conductivity, four points probe measurements were performed. These measurements were focused on measuring the sheet resistance only, since it is very well know that electrical conductivity through UNCD films is controlled extensively by the grain boundaries [22, 23], due to dangling bonds, and over the surface by quantum states of holes accumulated on the 2D surface layer formed on hydrogenated air-exposed top-surface region of diamond $[11,12]$. By comparing the sheet resistance results with the Raman analysis, it can be seen that those films that have low sheet resistance ( $\mathrm{m} \Omega$ to $\Omega$ ) 
correspond to those featuring the graphite phase only or UNCD films with $\mathrm{sp}^{2}$ hybridization bonds in the grain boundaries (confirmed by HRTEM analysis). On the other hand, films with high sheet resistance ( $\mathrm{k} \Omega$ to $\mathrm{M} \Omega$ ) correspond to those with more TP-A and more organized structure between grain boundaries (see HRTEM results). Therefore, it is relevant to optimize the structure of the UNCD films, in terms of graphite-diamond phases, which according to the results presented above can be achieved by optimizing the filament-substrate distance and the substrate surface temperature. The experimental evidence shown in this paper, indicates that being able to change the sheet resistance of the UNCD films may provide the bases for application of these films to Schottky and/or thin film transistor devices, since hydrogenated bulk diamond and polycrystalline diamond (MCD) have been used as active layer for those kind of devices [24-27].The current problem with yet non-optimized UNCD films relates to the low surface resistance $(\mathrm{m} \Omega)$, while all the devices such as FETs and MISFET that have been fabricated with hydrogenated surface exhibit resistance in the order of $(\mathrm{k} \Omega-\mathrm{M} \Omega)$. However, it is expected that the sheet resistance of the UNCD films can be increased by tailoring the grain boundaries chemical bonds, as a promising option, which is currently being explored.

\section{Conclusions}

Systematic studies were performed to investigate the effect of different substrate-filaments distance, substrate surface temperature and precursor's reactions in the space between filaments and substrate, during the growth of Ultrananocrystalline Diamond (UNCD) films using the HFCVD process. Analysis of the grown films, using complementary (Raman, HRTEM, SEM and $\mathrm{XRD)}$ characterization techniques and sheet resistance measurements, revealed the important role played by C-based precursor's reaction at different traveling distance from the moment of being cracked on the surface of the hot filaments until nucleation on the substrate surface to produce 
films with different structures, including structural disordered graphene, UNCD-Graphite mixture or single phase UNCD. Films grown at $5 \mathrm{~mm}$ filament-substrate distance show graphite and structural disordered graphene phases. Films grown at 15 and $30 \mathrm{~mm}$ filament-substrate distance show UNCD embedded in a graphite matrix structure, and films grown at 20, 25 and $35 \mathrm{~mm}$ filament-substrate distance show the characteristic UNCD structure, and grain boundaries with $\mathrm{sp}^{2}$ and TP-A chemical bonds configurations, respectively. Films with grain boundaries featuring mainly $\mathrm{sp}^{2}$ chemical bonds exhibit low sheet resistance $(\mathrm{m} \Omega-\Omega)$, while films featuring mainly TPA bonded boundaries show high sheet resistance $(\mathrm{k} \Omega-\mathrm{M} \Omega)$. The finding that UNCD films can also be grown at relatively low temperature $\left(\sim 490{ }^{\circ} \mathrm{C}\right)$, using an $\mathrm{Ar} / \mathrm{CH}_{4} / \mathrm{H}_{2}$ gas mixture and without using unconventional gases such as $\mathrm{H}_{2} \mathrm{~S}$ used in the past [6] to grow diamond films at low temperature by HFCVD, correlates with prior demonstration that UNCD films can be grown at temperatures in the range $350-400{ }^{\circ} \mathrm{C}$ via the MPCVD process [1], and has important implications for HFCVD growth of UNCD films for application to fabrication of Thin Film Transistors (TFTs) or Schottky diodes with hydrogenated surface for electronic power devices.

\section{Acknowledgments}

O. Auciello acknowledges support from the University of Texas Dallas through his Distinguished Endowed Chair Professor position.

M.J. Yacamán acknowledges support from the National Center for Research Resources (grant 5

G12RR013646-12) and the National Institute on Minority Health and Health Disparities (grant G12MD007591) from the National Institutes of Health and the Welch Foundation (grant No. AX$1615)$.

\section{References}


1. O. Auciello and A.V. Sumant, Status review of the science and technology of ultrananocrystalline diamond (UNCD ${ }^{\mathrm{TM}}$ ) films and application to multifunctional devices, Diamond and Related Materials, 19(7-9) (2010) 699.

2. X. Xiao, J. Birrell, J.E. Gerbi, O. Auciello and J.A. Carlisle, Low temperature growth of ultrananocrystalline diamond, J. of Appl. Phys., 96(4) (2004) 2232.

3. A.R. Krauss, O. Auciello, D.M. Gruen, A. Jayatissa, A. Sumant, J. Tucek, D. Mancini, N. Moldovan, A. Erdemir, D. Ersoy, M.N. Gardos, H.G. Busmann, E.M. Meyer, and M.Q. Ding, Ultrananocrystalline diamond thin films for MEMS and moving mechanical assembly device, Diamond and Related Materials, 10(11) (2001) 1952.

4. O. Auciello, J. Birrell, J.A. Carlisle, J.E. Gerbi, X. Xiao, B. Peng and H.D. Espinosa, Materials science and fabrication processes for a new MEMS technology based on ultrananocrystalline diamond thin films, J. of Physics: Condensed Matter, 16(16) (2004) R539.

5. J. Zimmer and K.V. Ravi, Aspect of scaling CVD diamond reactors, Diamond Related Materials 15 (2006) 229.

6. F. Piazza and G. Morell, Synthesis of diamond at sub $300{ }^{\circ} \mathrm{C}$ substrate temperature, Diamond and Related Materials 16 (2007)1950.

7. E.M.A. Fuentes-Fernandez, J.J. Alcantar-Peña, G. Lee, A. Boulom, H. Phan, B. Smith, T. Nguyen, S. Sahoo, F. Ruiz-Zepeda, M.J. Arellano-Jimenez, Pablo Gurman, C.A. MartinezPerez, M.J. Yacaman, R.S. Katiyar, and O. Auciello, Synthesis and characterization of microcrystalline diamond to ultrananocrystalline diamond films via Hot Filament Chemical Vapor Deposition for scaling to large area applications, Thin Solid Films, 603 (2016) 62.

8. X. Xiao, J. Wang, J. A. Carlisle, B. Mech, R. Greenberg, R. Freda, M. S. Humayun, J. Weiland, and $\mathrm{O}$. Auciello, In vitro and in vivo evaluation of ultrananocrystalline diamond for coating of implantable retinal microchips J. Biomed. Mater. Res. B, Applied Biomaterials, 77(2) 273-81 (2006).

9. O. Auciello, P. Gurman, M. B. Guglielmotti, D.G. Olmedo, A. Berra and M. J. Saravia, Biocompatible ultrananocrystalline diamond coatings for implantable medical devices, MRS Bulletin, vol. 39 (07) (2014) 621.

10. P. Bajaj, D. Akin, A. Gupta, D. Sherman, B. Shi, O. Auciello, R. Bashir, Ultrananocrystalline diamond film as an optimal cell interface for biomedical applications, Biomed. Microdevices, 9(6) (2007) 787.

11. L. Gan, E. Baskin, C. Saguy, and R. Kalish, Quantization of 2D Hole Gas in Conductive Hydrogenated Diamond Surfaces Observed by Electron Field Emission, Phys. Rev. Lett., 96(19) (2006) 196808.

12. C.E. Nebel, B. Rezek, and A. Zrenner, Electronic properties of the 2D-hole accumulation layer on hydrogen terminated diamond, Diamond and Related Materials, 13(11-12) (2004) 2031.

13. L. Gan, C. Saguy, E. Baskin, and R. Kalish, Electron field emission from the $2 D$ hole gas in hydrogen terminated, polycrystalline diamond, Diamond and Related Materials 17(3) (2008) 336.

14. V.I. Merkulov, J. S. Lannin, C. H. Munro, S. A. Asher, V. S. Veerasamy, and W. I. Milne, $u v$ Studies of Tetrahedral Bonding in Diamondlike Amorphous Carbon, Phys. Rev. Lett., 78(25) (1997) 4869.

15. J. Robertson and E.P. O'Reilly, Electronic and atomic structure of amorphous carbon, Phys. Rev. B, 35(6) (1987) 2946.

16. F. Klauser, D. Steinmüller-Nethl, R. Kaindl, E. Bertel and N. Memmel, Raman Studies of Nanoand Ultra-nanocrystalline Diamond Films Grown by Hot-Filament CVD, Chemical Vapor Deposition,. 16(4-6) (2010) 127. 
17. A.C. Ferrari and J. Robertson, Interpretation of Raman spectra of disordered and amorphous carbon, Phys, Rev. B, 61(20) (2000) 14095.

18. F. Mendoza, T.B. Limbu, B.R. Weiner, and G. Morell, Large-area bilayer graphene synthesis in the hot filament chemical vapor deposition reactor, Diamond and Related Materials, 51 (2015) 34.

19. B.V. Deryagin and D.V. Fedosayev, Special Issue on Diamond Growth and Films The growth of diamond and graphite from the gas phase, Surface and Coatings Technology, 38(1) (1989) 131.

20. T. Evans and P.F. James, A Study of the Transformation of Diamond to Graphite, Proceedings of the Royal Society of London A: Mathematical, Physical and Engineering Sciences, 277(1369) (1964) 260.

21. Y. Fu, H. Du, and C.Q. Sun, Interfacal Structure, residual stress and adhesion of diamond coatings deposited on titanium, Thin Solid Films, 424(1) (2003) 107.

22. L. Wang, Q. Zeng, J. Huang, R. Xu, K. Tang, J. Zhang, Y. Xia, Influence of the diamond grain size on the electrical properties of nano-crystalline diamond film detectors, Thin Solid Films, 520(2) (2011) 717.

23. L. Gan, A. Bolker, C. Saguy, R. Kalish, D.L. Tan, B.K. Tay, D. Gruen, P. Bruno, The effect of grain boundaries and adsorbates on the electrical properties of hydrogenated ultra nano crystalline diamond, Diamond and Related Materials, 18(9) (2009) 1118.

24. J.W. Liu, M. Y. Liao, M. Imura, E. Watanabe, H. Oosato, and Y. Koide, Diamond logic inverter with enhancement-mode metal-insulator-semiconductor field effect transistor, Appl. Phys. Lett., 105(8) (2014) 082110.

25. J.C. Madaleno, S.C. Trippe, and L. Pereira, Comparison of the electrical behavior of Schottky diodes built on the nucleation and growth surfaces of polycrystalline diamond, Diamond and Related Materials, 16(4-7) (2007) 926.

26. H. Kozak, A, Kromka, O. Babchenko, and B. Rezek, Directly Grown Nanocrystalline Diamond Field-Effect Transistor Microstructures, Sensor Letters, 8(3) (2010) 482.

27. O.A.Williams, Ultrananocrystalline diamond for electronic applications, Semiconductor Science and Technology, 21(8) (2006) R49 